\title{
Spread deficits in initiation, speed and accuracy of horizontal and vertical automatic saccades in dementia with Lewy bodies
}

\author{
Zoi Kapoula ${ }^{*+}$, Qing Yang ${ }^{1 *}$, Marine Vernet ${ }^{1}$, Benedicte Dieudonné ${ }^{2}$, Sandrine Greffard ${ }^{2}$ and Marc Verny ${ }^{2}$ \\ ' Group IRIS, FRE 3375, Center National de la Recherche Scientifique, Service d'Ophtalmologie-ORL-Stomatologie, Hôpital Européen Georges Pompidou, Paris, France \\ 2 Service de gériatrie, Hôpital Pitie Salpétrière, Université Paris VI, Paris, France
}

Edited by:

Walter E. Kaufmann, Johns Hopkins

School of Medicine, USA

\section{Reviewed by:}

Jose Biller, Loyola University Medical Center, USA

Thomas Eggert, Ludwig-Maximilians

Universität, Germany

\section{*Correspondence:}

Zoi Kapoula and Qing Yang, Group IRIS, Vision and Motricité Binoculaire, FRE 3375, Center National de la Recherche Scientifique/International Standard Book Number, Service d'Ophtalmologie-ORL-Stomatologie, Hôpital Européen Georges Pompidou, 20 rue Leblanc, Paris 75015, France.

e-mail:zoi.kapoula@egp.aphp.fr; qing. yang@egp.aphp.fr

'Zoi Kapoula and Qing Yang contributed equally to this work.

\begin{abstract}
Background: Mosimann et al. (2005) reported prolongation of saccade latency of prosaccades in dementia with Lewy body (DLB). The goal of this study is to go further examining all parameters, such as rates of express latency, but also accuracy and velocity of saccades, and their variability. Methods: We examined horizontal and vertical saccades in 10 healthy elderly subjects and 10 patients with DLB. Two tasks were used: the gap (fixation target extinguishes prior to target onset) and the overlap (fixation stays on after target onset). Eye movements were recorded with the Eyelink II eye tracker. Results: The main findings were: (1) as for healthy, latencies were shorter in the gap than in the overlap task (a gap effect); (2) for both tasks latency of saccades was longer for DLB patients and for all directions; (3) express latency in the gap task was absent for large majority of DLB patients while such saccades occurred frequency for controls; (4) accuracy and peak velocity were lower in DLB patients; (5) variability of all parameters was abnormally high in DLB patients. Conclusions: Abnormalities of all parameters, latency, accuracy and peak velocity reflect spread deficits in cortical-subcortical circuits involved in the triggering and execution of saccades.
\end{abstract}

Keywords: Lewy body, saccades, latency, accuracy, velocity, variability

\section{INTRODUCTION}

Dementia with Lewy bodies (DLB) is characterized by dementia, relatively mild parkinsonism, visual hallucinations, and fluctuations in conscious level. Unlike Alzheimer's disease (AD), DLB shows cortical-subcortical degeneration (Horoupian and Wasserstein, 1999), which can affect both initiation and execution of eye movements. The abnormal cytoplasmic inclusions that develop in DLB are very similar to those observed in Pakinson's disease (PD). Symptoms and pathophysiology of DLB overlaps also with that of AD. Mosimann et al. (2005) used gap and overlap saccade tasks in $\mathrm{DLB}, \mathrm{AD}, \mathrm{PD}$, and age-matched controls; in the former task the fixation point extinguishes $200 \mathrm{~ms}$ before target onset while in the latter fixation point stays on during the target presentation. The gap task usually elicits shorter latency saccades, some of them being extra short (80-120 ms); they are called express saccades. Mosimann et al. (2005) reported long latency in DLB patients even in the gap task. In contrast, $\mathrm{AD}$ and PD patients made normal short latency saccades in the same task. For more voluntary saccades, e.g., predictive saccades, antisaccades, all groups of patients (DLB, $\mathrm{AD}$, and PD) showed abnormally long latencies. Mosimann et al. (2005) emphasized the need for further research on eye movements as such studies may have a potential for differential diagnosis for these diseases. The study is a part of a long term program aiming to provide a complete characterization of saccades in DLB, AD patients and controls (national PHRC). As a first step, here we report a study of saccades in the gap and overlap tasks in a group of DLB patients and age-matched controls. It examines saccades for both horizontal and vertical directions, and the occurrence of express saccades in the gap task versus overlap in healthy and DLB patients. In our laboratory normal rates of express saccades, about $20 \%$, were observed for both young and elderly subjects (Yang and Kapoula, 2006; Yang et al., 2006). For PD patients, however, the results of express saccades are controversial. Armstrong et al. (2002) and van Stockum et al. (2008) reported more express in PD patients than in healthy subjects while other studies reported less express (Ratmanov et al., 2006) or no difference (Roll et al., 1996). Different cortical-subcortical areas, such as, superior colliculus (Isa and Kobayashi, 2004), posterior parietal cortex (Kapoula et al., 2001, 2004), or frontal eye field (Kurkin et al., 2003) could be involved in the generation saccades. Triggering of express type of saccades is believed to involve a shorter circuit, e.g., retino-superior colliculus, or retino-occipital-parietal-superior colliculus, while triggering of voluntary saccades involves a more extended circuit including the frontal lobe (Pierrot-Deseilligny et al., 1995, 2002).

The second goal of this study is to examine accuracy and speed characteristics of saccades. Saccade velocity depends mainly on function of brainstem structures (Paramedian Pontine reticular Formation - PPRF for horizontal saccades, the rostral interstitial nucleus of medial longitudinal fasciculus - riMLF for vertical); accuracy of saccades depends on both cortical and subcortical areas (Leigh and Zee, 2006). The metrics and dynamics of saccadic eye movements have not yet been studied in patients with 
DLB. In the literature, the effect of normal aging on the accuracy of saccades is controversial. For horizontal saccades in healthy elderly, some studies (Sharpe and Zackon, 1987; Tedeschi et al., 1989) reported a significant increase in the percentage of hypometric amplitude, especially for targets eccentricities larger than $20^{\circ}$ (Irving et al., 2006). However, many other studies (Warabi et al., 1984; Moschner and Baloh, 1994; Munoz et al., 1998) found no effect of aging on saccade metrics. A similar controversy exists for vertical saccades (Huaman and Sharpe, 1993; Yang and Kapoula, 2008). On the other hand, abnormality in accuracy of saccades has been found in patients with dementia, such as AD, frontotemporal dementia and semantic dementia (Shafiq-Antonacci et al., 2003; Garbutt et al., 2008).

Concerning the speed of saccades many studies described reduction in peak velocity in normal elderly relative to young subjects (Sharpe and Zackon, 1987; Tedeschi et al., 1989) and in patients with AD relative to healthy elderly (Shafiq-Antonacci et al., 2003). Yet, these reductions in peak velocity were most evident with large amplitude saccades, usually to targets beyond $20^{\circ}$ eccentricity. Note that in another study (Munoz et al., 1998), no aging effects were observed either on accuracy or on peak velocity (for target at $20^{\circ}$ of eccentricity). Thus whether saccade accuracy and speed are subject to aging is controversial. But there is evidence for their abnormalities in dementia. As DLB patients are reported to have both cortical and subcortical deficits we expect to find abnormalities in all parameters of saccades (latency, accuracy and speed).

Finally, patients with DLB are known to have cognitive performance with high fluctuation of attention. We wondered to what extent clinically assessed fluctuation is associated with variability in saccade parameters. A few previous studies (Munoz et al., 1998; Yang et al., 2002) found that variability of latency was significantly larger in children than in adults. More recently, the former group also reported high variability of saccade latency in elderly (Peltsch et al., 2009). High variability of saccade latency in studies with patients can point particular neural deficits. For instance, Blekher et al. (2006) showed that patients with Huntington's disease demonstrated increased error rate, increased mean latency and increased variability of latency for memory-guided saccades and antisaccades. Thus, the present study including variability of all parameters allows complete characterization of saccade performance in DLB patients.

\section{MATERIALS AND METHODS SUBJECTS}

Ten healthy subjects aged between 65 and 88 years (mean $78.5 \pm 6.8$ ) were recruited either among senior laboratory workers, or in the ophthalmology service in the hospital, coming for routine examination and spectacle correction. They had corrected visual acuity above $5 / 10$. The cognitive performance as measured by Mini Mental State Examination (Folstein et al., 1975) was more than 28 of 30 points. No subject showed visual, neurological, psychiatric disorders or received medication with neural system effects. Binocular vision was assessed with TNO test for stereoscopic vision (Test designed by the Netherlands Organization for Applied Scientific Research - TNO); all individual scores were normal, 60' of arc or better.
Nineteen patients were recruited from the geriatric service in the hospital Pitié Salpêtrière (Paris). Recruitment was done using the consensus guidelines to diagnose DLB. All DLB patients had progressive cognitive decline of sufficient magnitude to interfere with normal social or occupational function and at least two following signs: fluctuating cognition with pronounced variation in attention and alertness, visual hallucinations and spontaneous features of parkinsonism. Moreover, DLB patients were different from Alzheimer patients by their neuropsychological characteristics (sub corticofrontal memory profile, visuo-spatial deficits, executive syndrome). They were different from Parkinson's disease because cognitive decline appeared before or at the same time as parkinsonism. Subjects with other cerebral pathology (e.g., stroke or significant white matter changes) were excluded by cerebral CT or MRI. All subjects with known history of macular degeneration or coexisting medical illness that could interfere with cognitive or visual testing were excluded. For 4 of 19 subjects no eye movement recording was possible because of recording difficulty or refusing the task. Ten patients with DLB completed all the oculomotor tests. Their ages were from 73 to 89 years (mean $81.6 \pm 4.7$ years). MMSE, Instrumental activities of daily living (Israêl, 1996), Dementia Rating Scale (Mattis, 1988), Hoehn and Yahr (1967) Scale were used to assess functional, neuropsychiatric symptoms and fluctuation. Demographical and clinical characteristics of these groups were shown in Table 1. Eight subjects were under medication "Exelon" (Simard and van Reekum, 2004).

The investigation adhered to the tenets of the Declaration of Helsinki and was approved by the institutional human experimentation committee (CPP Pitié Salpêtrière). Informed consent was obtained from all subjects after the nature of the procedure had been explained.

\section{VISUAL DISPLAY}

The visual display, shown in Figure 1A, was composed by five white luminous dots (angular size $0.2^{\circ}$ ), presented on a black computer screen. One of these five dots was at the center of the screen, two were at an eccentricity of $\pm 10^{\circ}$ horizontally and two were at an eccentricity of $\pm 10^{\circ}$ vertically.

Table 1 | Demographical and clinical characteristics of the groups.

\begin{tabular}{lcc}
\hline & Control & DLB \\
\hline$n$ & 10 & 10 \\
Age (years) & $78.2 \pm 4.6$ & $81.6 \pm 4.7$ \\
Education (years) & $13 \pm 2.1$ & $12.1 \pm 3.4$ \\
Estimated dementia & $\mathrm{NA}$ & $5.1 \pm 2.5$ \\
duration (years) & & \\
MMSE (max. 30) & $28.7 \pm 0.8$ & $22 \pm 4.6$ \\
Mattis & & $114 \pm 12$ \\
IADL & & $2.7 \pm 1.0$ \\
Hoehn and Yahr scale & $\mathrm{NA}$ & $1.6 \pm 1.0$ \\
Fluctuation & & $(+/+++)$ \\
\hline
\end{tabular}

MMSE - Mini mental state examination (max. = normal); Mattis - Mattise dementia rating scale (max. $144=$ normal); $I A D L$ - Instrumental activities of daily living (max. 4 = normal); Hoehn and Yahr scale - symptoms of Parkinson's disease progress (max. 5, $0=$ normal). 


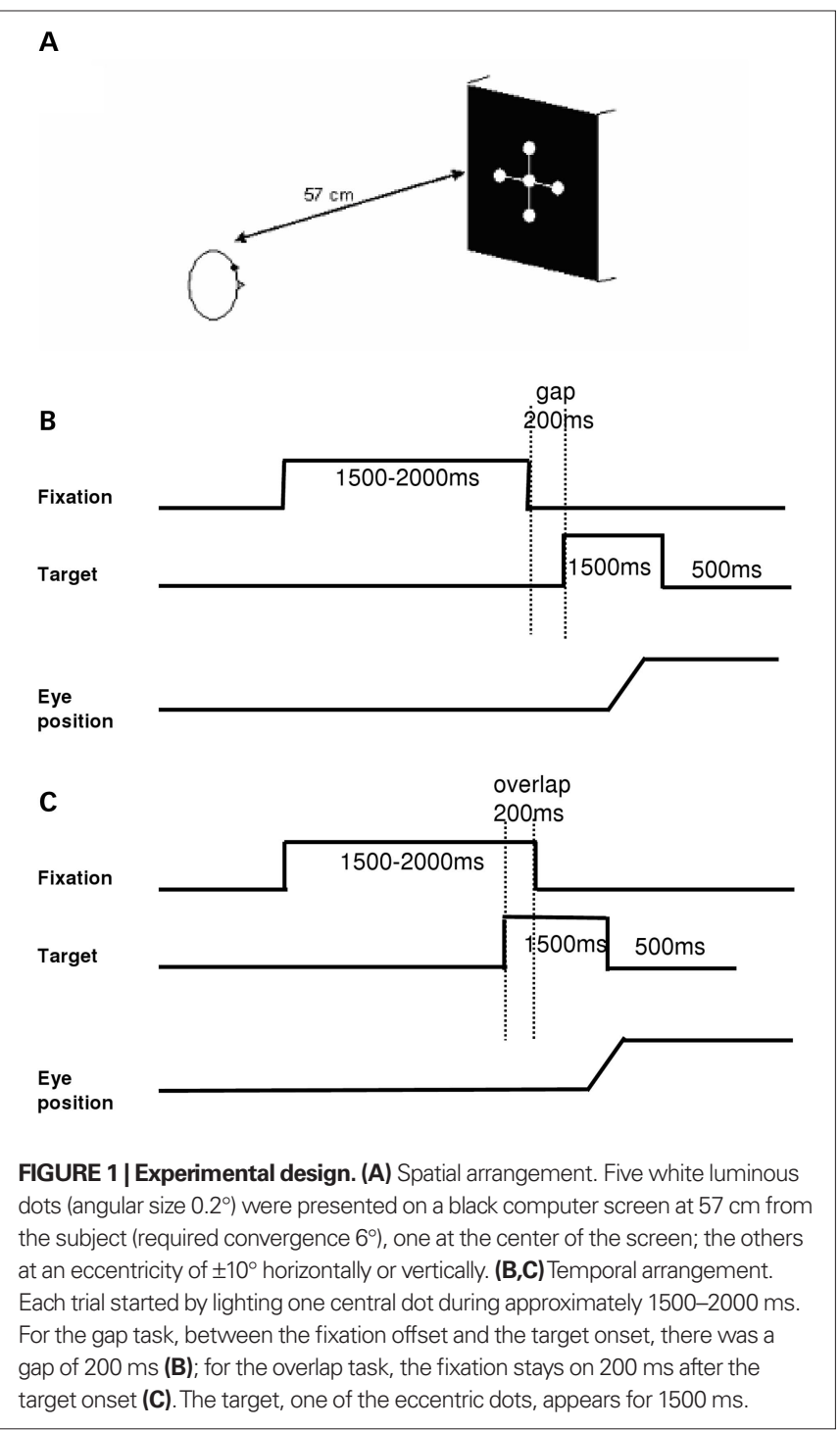

The subject was comfortably seated in an adapted chair, his head resting on a chin and frontal support. The subject viewed binocularly; all dots were highly visible.

\section{FIXATION AND OCULOMOTOR TASKS: GAP AND OVERLAP TASKS}

Each trial started by lighting a fixation dot at the center. The fixation dot stayed on for a random period between 1.5 and $2 \mathrm{~s}$. In the gap task, there was a time interval of $200 \mathrm{~ms}$ between the offset of the fixation point and the onset of the saccade target. The target dot was kept on for $1.5 \mathrm{~s}$ (Figure 1B). In the overlap task, the fixation point remained illuminated for $200 \mathrm{~ms}$ after the target dot appeared. The target stayed on also for another $1.5 \mathrm{~s}$ (Figure 1C). Subjects were required to make a saccade to the target point as rapidly and accurately as possible. A period of complete darkness of $500 \mathrm{~ms}$ was for break. Subjects were instructed to use this period for blinks. The total mean length of each trial was about $4 \mathrm{~s}$. In each block only gap or overlap task was used randomly for four directions, targets at $10^{\circ}$, left or right, up or down (10 trails for each direction, total 40 trials). One block for each task lasted 4-5 min. Total duration of the recording was about $10 \mathrm{~min}$.
A calibration sequence was performed at the beginning; the target made the following predictive sequence for each viewing distance: center, $10^{\circ}$ to left, center, $10^{\circ}$ to right, center, $10^{\circ}$ to up, center, $10^{\circ}$ to down, center; the target stayed at each location for $2 \mathrm{~s}$. From these recordings we extracted calibration factors.

\section{EYE MOVEMENT RECORDING}

Horizontal and vertical eye movements were recorded binocularly with the EyeLink II device. Each channel was sampled at $250 \mathrm{~Hz}$. The system has a spatial resolution of $0.025^{\circ}$ in pupil-CR mode and saccade event resolution of $0.05^{\circ}$ for microsaccades.

\section{DATA ANALYSIS}

From the two individual calibrated eye position signals we derived the conjugate signal (left eye + right eye)/2. The onset and the offset of saccades were defined as the time when conjugate eye velocity exceeded or dropped below $10 \%$ of the peak velocity. The process was performed automatically by the computer, and the verification was made by visual inspection of the individual eye position and velocity traces. For both gap and overlap tasks latency was measured as the time between target onset (time 0) and saccade onset (mark "i" in Figure 2). To estimate the accuracy we used two measures: first, the amplitude of primary main saccade (between "i" and "p") relative to the target eccentricity (gain = saccade amplitude/target eccentricity); second the amplitude of the final eye position (between " $i$ " and "f") after execution of one or more corrective saccades relative to the target eccentricity (see Figure 2 ). Peak velocity of the primary saccade were also analyzed. To evaluate the variability of each parameter, we calculated the coefficient of variation $(\mathrm{CV})$, the relative standard deviation expressed as a unitless proportion of each subjects mean (van Beers, 2007; Peltsch et al., 2009).

Eye movements in the wrong direction, with latency shorter than $80 \mathrm{~ms}$ (anticipation) or longer than $1500 \mathrm{~ms}$, or contaminated by blinks were rejected. For healthy elderly $9 \%$ of trials (ranged from $4 \%$ to $13 \%$ ) and for patients with DLB $14 \%$ of trials (from $2 \%$ to $25 \%$ ) had to be rejected, the most frequent reason being the blinks. Express latencies were defined as those falling in the range from 80 to $120 \mathrm{~ms}$; the rate of express saccades refers to the total number of valid saccades.

\section{STATISTICAL}

A three-way analysis of variance (ANOVA) was performed on individual mean latency with the between subjects factor - group (healthy, DLB) and the within-subjects factors - the oculomotor task (gap, overlap) and the direction (left, right, up and down). Two-way ANOVA was performed on individual mean accuracy and velocity means with the between subjects factor (healthy, DLB) and the within-subjects factor (saccade direction). Post hoc comparisons were done with the Least Significant Differences test. For the rates of express latency and for the CV of each parameter, the non-parametric Mann-Whitney $U$-test was used for comparisons between healthy elderly and patients with DLB; for withinsubjects comparisons between different directions the Friedman and Wilcoxon test was used. 


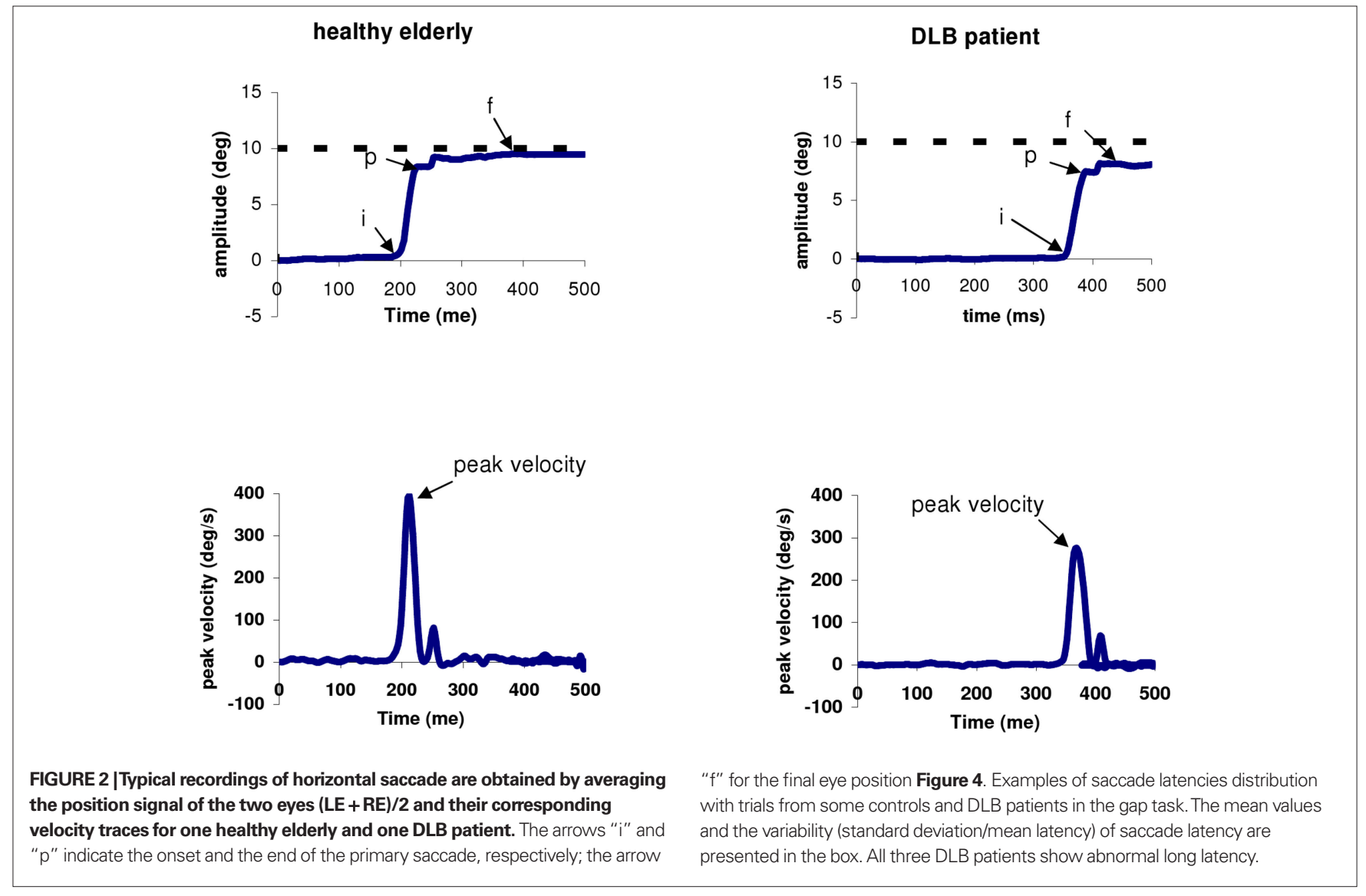

\section{RESULTS}

\section{LATENCY}

\section{Mean saccade latency}

Figure 3 shows group mean latencies of saccades with standard error (SE) for different directions under the gap (a) and the overlap (b) tasks in healthy elderly and patients with DLB. The three-way ANOVA showed a group effect, i.e., significantly longer latencies for patients with DLB than for healthy elderly $\left(F_{1,18}=11.83, p<0.01\right)$ and a gap effect, i.e., significantly shorter latencies for gap task than for overlap task $\left(F_{1,18}=47.21\right.$, $p<0.01)$, but no direction effect $\left(F_{3,51}=0.53, p=0.66\right)$. Further post hoc comparisons showed that the group effect was significant for both gap and overlap tasks for all four directions (all $p<0.05$ ); the mean values of group effect were $164 \mathrm{~ms}$ (SD $21 \mathrm{~ms}$ ) and $176 \mathrm{~ms}$ (SD $46 \mathrm{~ms}$ ) for gap and overlap tasks, respectively. The gap effect was significant for all directions and for both groups of elderly (all $p<0.001$ ). The mean values of gap effect were 105 and $118 \mathrm{~ms}$ for healthy elderly and patients with DLB, respectively.

\section{Variability in saccade latency}

Figure 4 shows some individual examples of latency variability. Latencies are displayed over successive trials in the gap task. The healthy subjects produce almost similar latency from one trial to next. In contrast, large fluctuations of latency are seen for the DLB subjects.
Figure 5 shows group mean CV in latency for different direction under the gap (a) and the overlap (b) conditions in healthy elderly and patients with DLB. The Mann-Whitney $U$-test showed the group effect, i.e., significantly high CV in latency for DLB patients than for healthy elderly for all directions (all $U<15, p<0.01$ ).

\section{Rates of express saccades}

Table 2 shows individual rate of express latency in the gap task for each direction. In the gap task substantial number of express latency was found. Nine of 10 healthy elderly subjects showed express saccades (rang between 7\% and 70\%); note the large individual differences. In contrast, only 3 of 10 DLB patients showed express latency. The mean rates for healthy elderly were $20.7 \%, 19.7 \%, 11.4 \%$, and $12.1 \%$ for leftward, rightward, upward, and downward, respectively; for patients with DLB mean rates were only $3.4 \%, 2.8 \% 2.4 \%$, and $0 \%$ for each direction, respectively. The Mann-Whitney $U$-test showed the group effect, i.e., significantly lower rates of express latency for patients with DLB than for control elderly (all $U=<23, p<0.05$ ).

\section{ACCURACY-SPEED}

ANOVA applied on the accuracy and speed data showed no significant difference between the gap and the overlap tasks (all $p>0.05$ ). Consequently, accuracy of the primary saccade and of the final eye position, and peak velocity of the primary saccade were regrouped from the two tasks; these grouped results will be presented below. 

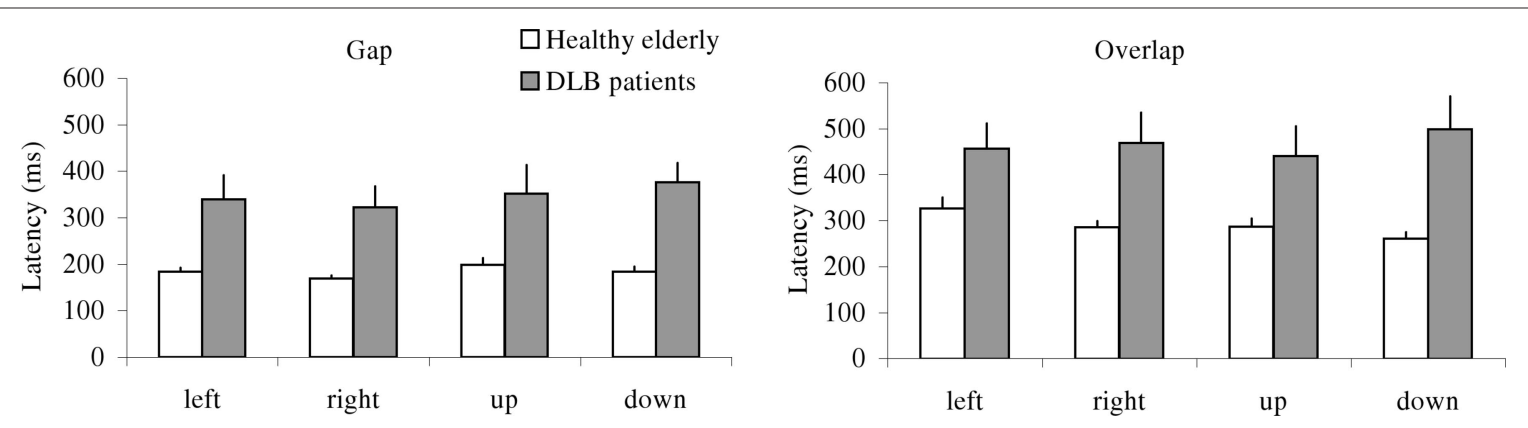

FIGURE 3 | Group mean latency of saccades with standard error in gap and overlap tasks, for four directions in healthy elderly and DLB patients; longer latencies of saccades for DLB patients than for healthy elderly for each condition.
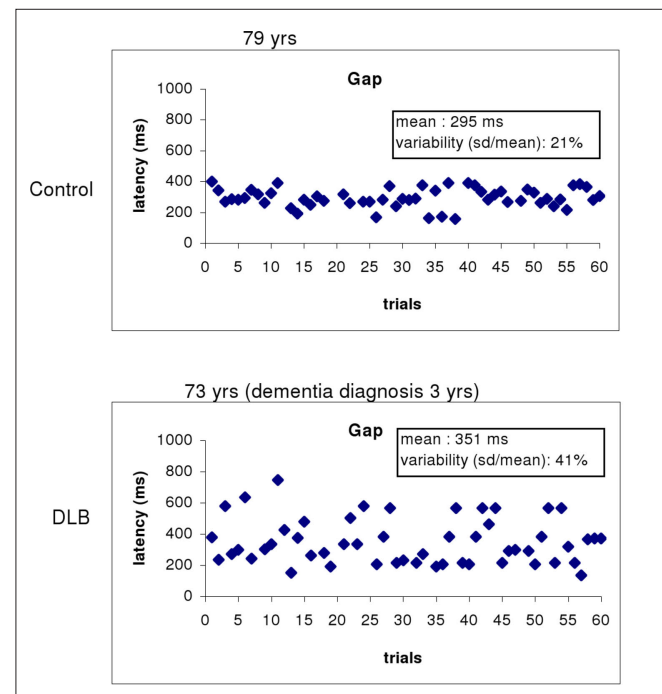

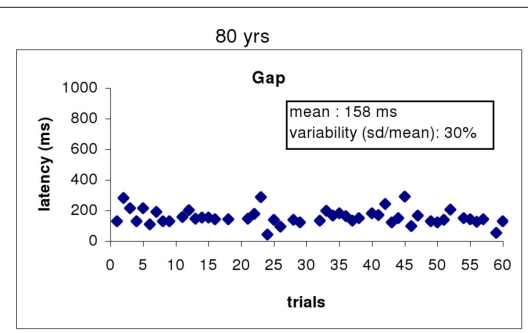

79 yrs (dementia diagnosis 4 yrs)

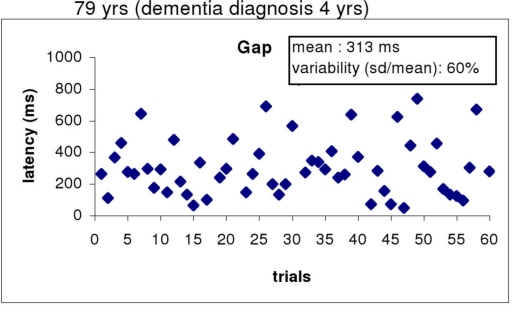

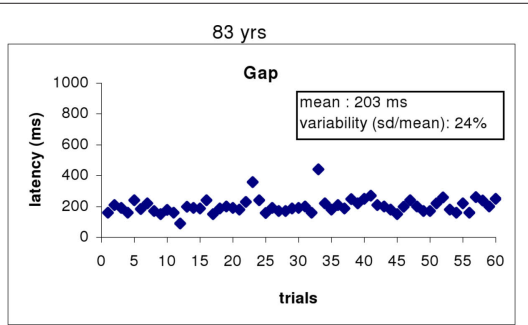

$81 \mathrm{yrs}$ (dementia diagnosis $8 \mathrm{yrs}$ )

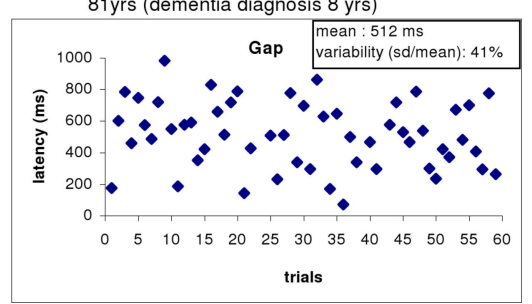

FIGURE 4 | Examples of saccade latencies distribution with trials from some controls and DLB patients in the gap task. The mean values and the variability (standard deviation/mean latency) of saccade latency are presented in the box. All three DLB patients show abnormal long latency.

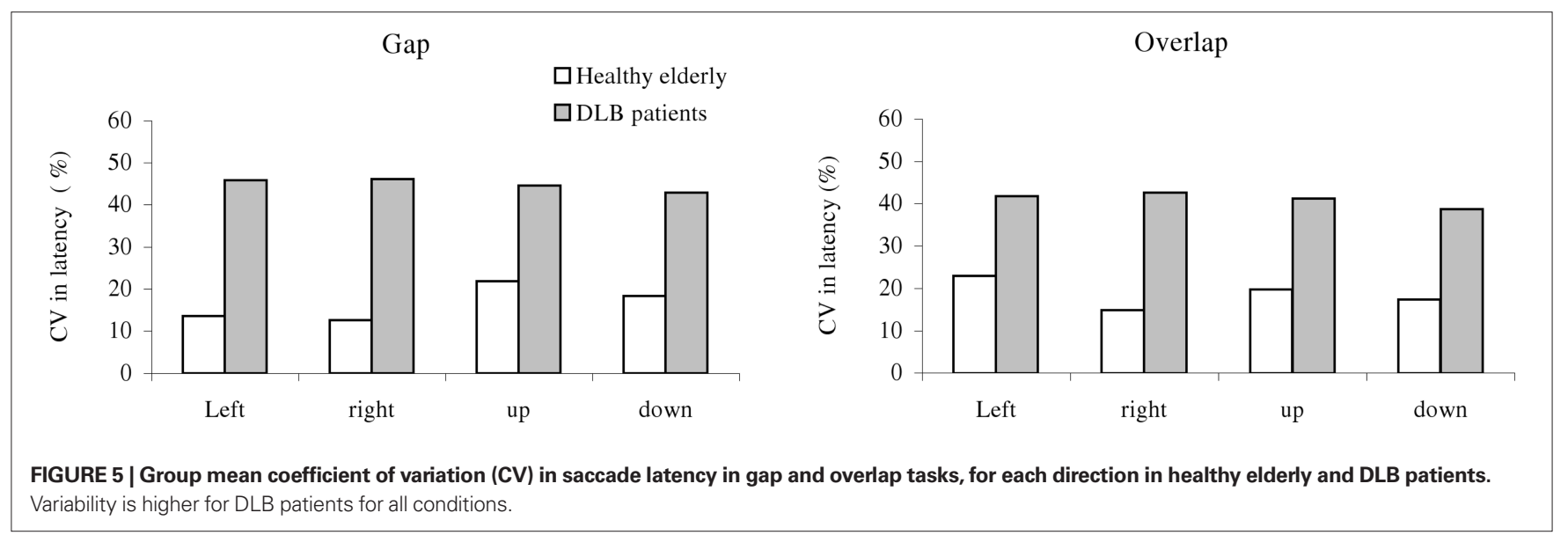

\section{Accuracy: gain of saccades}

Figure 6 shows group mean gain of the primary saccade and of the final eye position together with SE; results are shown for different directions in healthy elderly and patients with DLB. The two-way ANOVA showed a group effect, i.e., significantly lower gain for patients than for healthy; this was the case for both the primary saccade $\left(F_{1,18}=15.63, p<0.01\right)$ and the final eye position $\left(F_{1,18}=11.28, p<0.01\right)$. Further post hoc comparisons showed that 
Table 2 | Rates of express saccades for controls and DLB patients.

\begin{tabular}{lrrrr}
\hline Subjects & Leftward & Rightward & Upward & Downward \\
\hline Control 1 & 0 & 0 & 0 & 0 \\
Control 2 & 16 & 43 & 7 & 8 \\
Control 3 & 0 & 0 & 7 & 7 \\
Control 4 & 25 & 25 & 15 & 8 \\
Control 5 & 20 & 18 & 12 & 0 \\
Control 6 & 38 & 25 & 8 & 0 \\
Control 7 & 25 & 20 & 8 & 43 \\
Control 8 & 0 & 0 & 11 & 0 \\
Control 9 & 13 & 16 & 31 & 47 \\
Control 10 & 70 & 50 & 15 & 8 \\
Mean control & 20.7 & 19.7 & 11.4 & 12.1 \\
DLB1 & 0 & 0 & 0 & 0 \\
DLB2 & 0 & 0 & 0 & 0 \\
DLB3 & 0 & 0 & 0 & 0 \\
DLB4 & 0 & 0 & 0 & 0 \\
DLB5 & 0 & 0 & 0 & 0 \\
DLB6 & 13 & 8 & 17 & 0 \\
DLB7 & 15 & 10 & 7 & 0 \\
DLB8 & 6 & 10 & 0 & 0 \\
DLB9 & 0 & 0 & 0 & 0 \\
DLB10 & 0 & 0 & 0 & 0 \\
Mean DLB & 3.4 & 2.8 & 2.4 & 0 \\
\hline & & & &
\end{tabular}

the group effect was significant for all directions (all $p<0.001)$. A direction effect was observed for the final eye position $\left(F_{3,54}=5.08\right.$, $p<0.01$ ), i.e., the gain was higher for horizontal saccades (both leftward and rightward) than for vertical saccades (upward or downward, all $p<0.05$ ).

\section{Variability in gain}

Figure 7 shows the group mean CV for the measured gain from the primary saccade and the final eye position in healthy and in patients. The Mann-Whitney $U$-test showed a group effect, i.e., significantly higher $\mathrm{CV}$ in gain for patients than for healthy for all directions and for both accuracy measures (all $U<19, p<0.05$ ).

\section{Velocity}

Figure 8 shows group mean peak velocity of the primary saccade with SE for different directions in healthy and in patients. The twoway ANOVA showed a group effect on peak velocity, i.e., significantly lower peak velocity for patients than for healthy $\left(F_{1,18}=7.45\right.$, $p<0.01)$. Further post hoc comparisons showed that the group effect on peak velocity was significant for all directions (all $p<0.001$ ).

\section{Variability in velocity}

Figure 9 shows group mean CV from peak velocity for each direction in healthy and patients. The Mann-Whitney $U$-test showed a group effect, i.e., significantly higher variability for DLB patients than for healthy (all $U<19, p<0.05$ ).

As the peak velocity of saccades increases with saccade amplitude increase lower peak velocity for DLB patients could be accounted by lower amplitude of saccades. Therefore, we selected saccades of similar amplitudes (between $9^{\circ}$ and $11^{\circ}$ ), e.g., the most accurate saccades relative to the target eccentricity $\left(10^{\circ}\right)$, and examined peak velocity and variability locally for this subgroup (see Figure 10). One-way ANOVA showed no significant difference of mean peak velocity between healthy and patients $\left(F_{1,18}=2.1, p=0.16\right)$. Yet, DLB patients showed higher local variability of peak velocity than healthy elderly $(U=20, p<0.05)$.

\section{DISCUSSION}

The main findings are: (1) abnormal long latency of saccades in gap and overlap tasks for DLB relative to healthy elderly occurs for all directions; (2) yet, similar gap effect (shorter latencies in gap than in overlap) exists for healthy and patients; (3) express latencies are absent or minimal in patients, but frequent in healthy; (4) accuracy and peak velocity are lower in DLB patients; (5) variability of all saccade parameters is abnormally high in DLB patients. These results will be discussed below.

\section{LONG LATENCIES FOR DLB FOR ALL DIRECTIONS}

Our results extend those of horizontal saccades from Mosimann et al. (2005) to vertical saccades showing longer latencies in DLB patients. Abnormally long latencies for all directions reflect dysfunction of common cortical oculomotor areas. The saccade-related activity of the SC neurons is shaped by inputs from the posterior parietal cortex, the frontal eye fields, and the substantia nigra pars reticulata, which control horizontal as well as vertical saccades (Leigh and Zee, 2006).

\section{GAP EFFECT}

The gap effect refers to the latency advantage of removing the fixation point before the onset of a target (Pratt et al., 1997). Such gap effect, shorter latency in the gap task than in the overlap task, was still existent in DLB (118 versus $105 \mathrm{~ms}$ in healthy). The percentages of latency decrease in the gap condition, (overlap-gap)/overlap, were similar in healthy elderly (26-42\%) and in DLB patients (21-36\%). These results are comparable with those reported in young subjects (20-41\%, Yang et al., 2006). What is the responsible factor for such gap effect is still controversial. The absence of the stimulus during the gap (Findlay and Walker, 1999), the offset of fixation (Ross and Ross, 1980, 1981; ReuterLorenz et al., 1991; Kingstone and Klein, 1993; Munoz et al., 1998) or advanced movement preparation (Rolfs and Vitu, 2007) could explain such phenomena. Although DLB patients showed longer latencies in both gap and overlap tasks than healthy elderly, the disengagement of fixation and perhaps attention was still facilitated by removal of the fixation point in the gap task for these patients.

\section{QUASI PAUCITY OF EXPRESS SACCADES}

Express latency is usually defined as the latency between 80 and $120 \mathrm{~ms}$. This is, however, arbitrary. Neurophysiological studies (Dorris and Munoz, 1995; Edelman and Keller, 1996; Sparks et al., 2000) have shown that express saccades are produced when visual and motor bursts in the SC merge to produce the saccade. In other words, the visual transient response in the SC caused by the appearance of the target becomes the direct motor command to trigger the saccade. It has been shown that this visual burst of activity in the SC must be added to an elevated baseline to cross the saccade threshold to trigger an express saccade (Dorris and Munoz, 1995; Everling et al., 1998). Changing the timing of the visual transient response by changing the intensity 

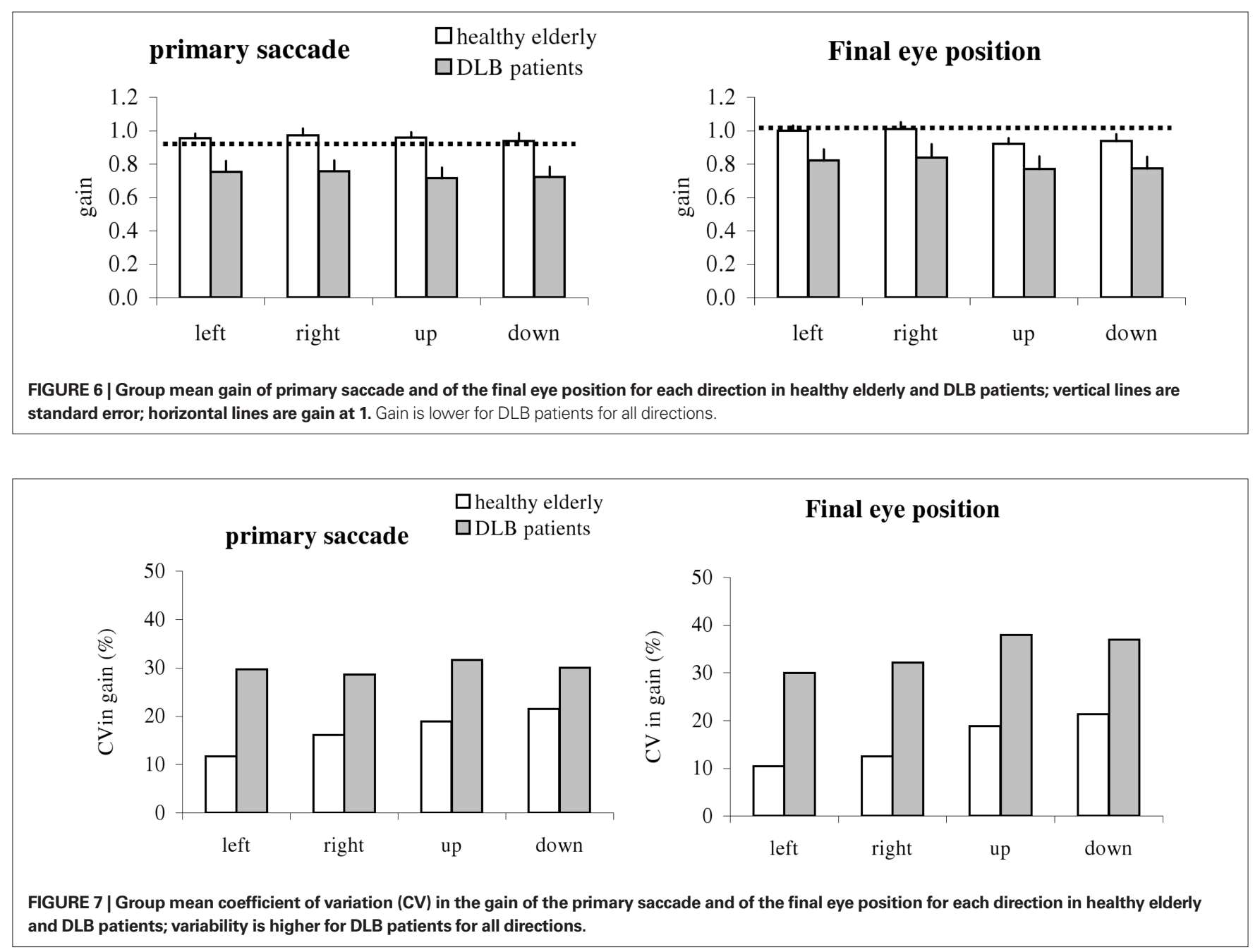

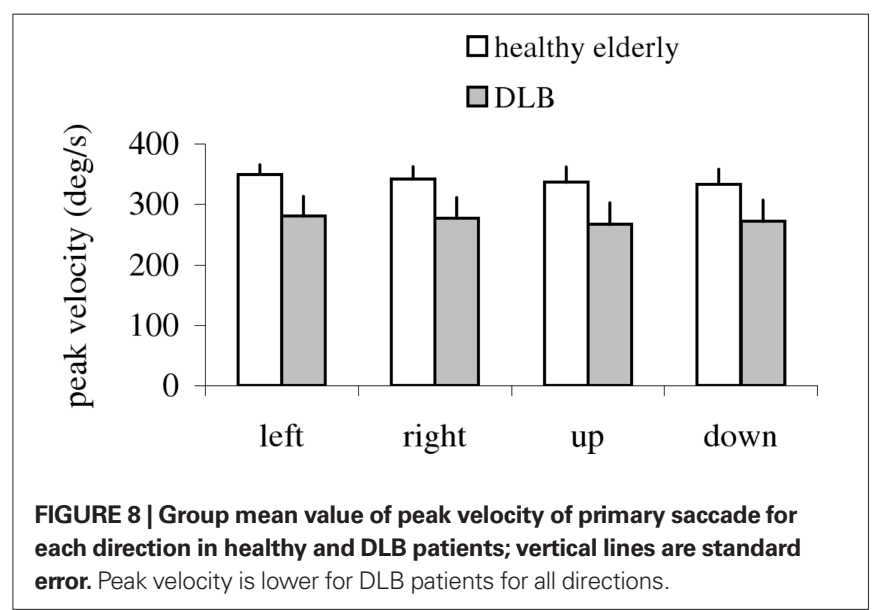

of the visual stimulus, leads to changes in when express saccades are triggered (Bell et al., 2000). Therefore, the express epoch could be specific in each laboratory. In the present study we compare healthy elderly and DLB patients using the same stimuli. Healthy elderly show similar rates of express latency as young adults (Yang and Kapoula, 2006; Yang et al., 2006). The decreased rates of express latency in DLB patients could suggest a deterioration of the hypothetical express

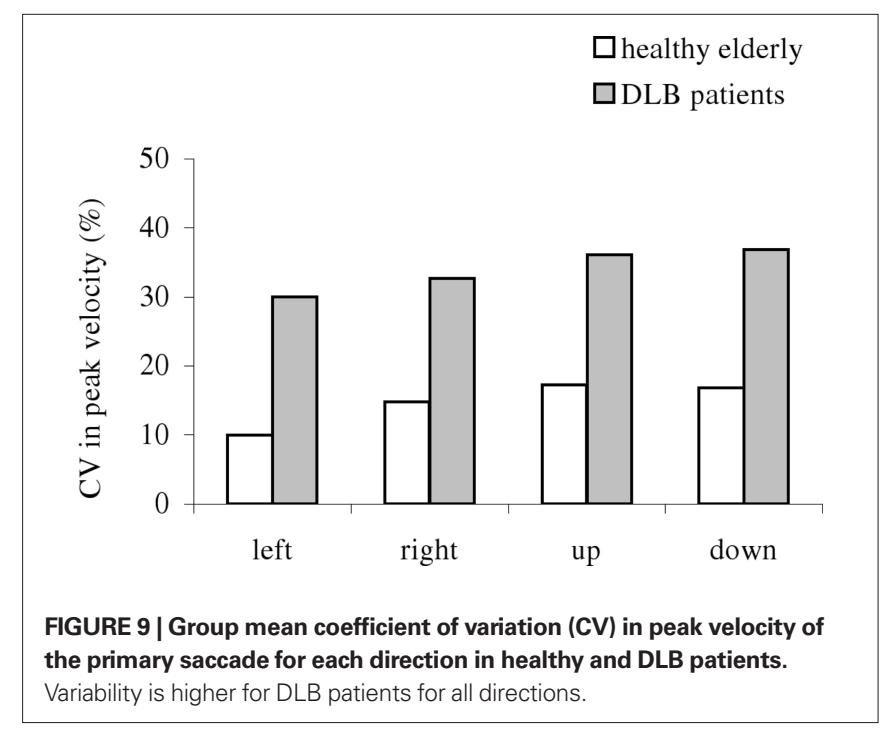

circuit and/or simply a delay in the visual transient response. Yet, the distribution of latencies (over two populations, see Figure 11) shows a multiple peak for healthy elderly, namely a peak at $110-120 \mathrm{~ms}, 160 \mathrm{~ms}$ and $190-200 \mathrm{~ms}$ while such peak is seen at 150 and $290 \mathrm{~ms}$ in DLB 
patients. In addition to the shift of the peaks, DLB patients showed spread distribution all over the scale. Thus, it is likely that the express circuit, usually attributed to the function of pathways including the parietal saccade regions and the superior colliculus is also impaired in DLB patients (de Bruin et al., 1992).

\section{LOWER GAIN AND PEAK VELOCITY}

DLB patients showed lower accuracy and peak velocity than control elderly. This is consistent with results in subjects with brainstem lesions. For example, subjects with progressive supranuclear palsy, who have prominent atrophy of the brainstem oculomotor regions (Groschel et al., 2004; Boxer et al., 2006), displayed deficits in saccade velocity and accuracy. In addition, saccade gain and peak velocity were reduced in adult patients with Niemann-Pick disease, a disorder known to affect brainstem and frontal cortical function (Abel et al., 2009). Importantly, in our study the deficits are present for all directions suggesting spread degeneration of both horizontal and vertical saccade generators located in the brainstem. Taken together, when cortical and subcortical neurodegeneration coexists, like in DLB patients, all parameters of reflexive saccades, such as latency, gain, velocity and their variability could be affected.

\section{HIGH VARIABILITY}

Saccade latency is directly linked to the neural functioning of oculomotor areas, e.g., FEF, PPC (Pierrot-Deseilligny et al., 1995, 2002), SC (Isa and Kobayashi, 2004), the substantia nigra pars reticulata and

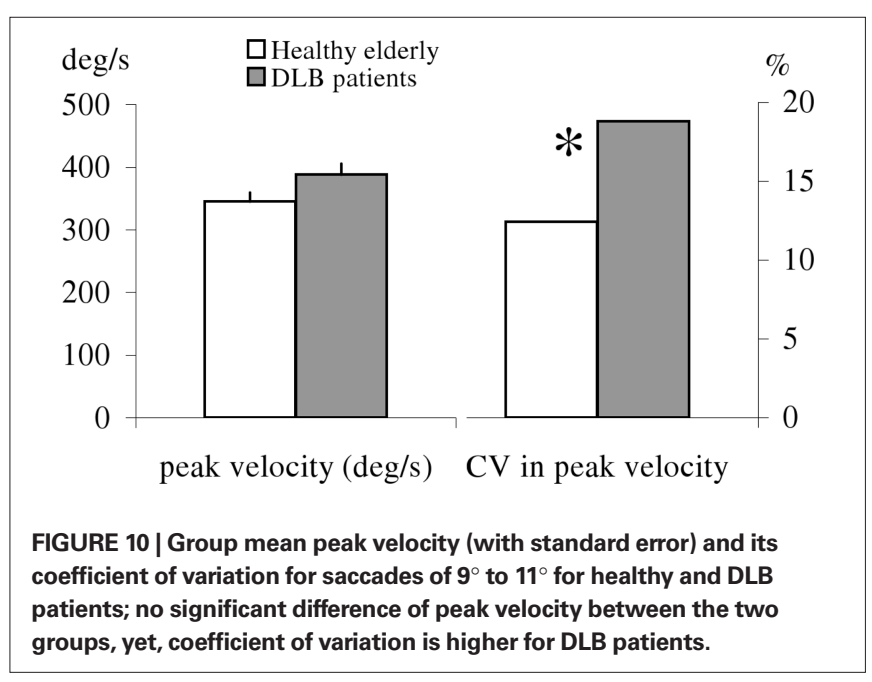

PPRF (Leigh and Zee, 2006). Studies of Hanes et al. (1998) showed that a class of movement-related neurons in the FEF have an activity that begins to rise before a saccade. The initiation of the saccade was associated with the activity reaching a fixed threshold and the rate of rise varies randomly from trial to trial. Importantly, the rate of rise was tightly correlated with the observed saccadic latency. A consequence of this observation is that the latency can be modified either by adjustment of the threshold, or by change of the rate of rise. It assumes that a collection of information is made at a certain rate (the rate of rise in the cells) until a threshold criterion is reached: this would cause a decision, in our case, a saccade. Manipulation of perception (Heywood and Churcher, 1980), decision criterion level (Reddi and Carpenter, 2000), or prior probability (Carpenter and Williams, 1995) leads to a change in latency, which could be explained by a change in the threshold. Manipulation of attention also strongly affects the latency, which could be explained by alteration of rate of rise (Heywood and Churcher, 1980). Previous studies (Munoz et al., 1998; Yang et al., 2002) found that variability of latency was significantly larger in children than in adults. This was attributed to poor control over visual fixation. The fixation control system involves a distributed cortical-subcortical circuit, e.g., parietal-frontal-superior colliculus (Fischer et al., 1995). Variability on latency depending on cortical structures is consistent with neuropsychological tests, e.g., indicating problems in executive functions and visual attention in DLB patients including working memory (McKeith et al., 2005). High variability for saccade metrics could also be related to function of parietal and frontal oculomotor areas providing the position signal error for the brainstem saccade generator (Leigh and Zee, 2006). For instance, variation in the representation of the saccade vector in the motor map of the SC could lead to variability in saccade accuracy (Van Opstal and Van Gisbergen, 1989). In addition, variability of accuracy can also be related to dysfunction at brainstem saccade generator itself and this is further corroborated by the variability observed on the peak velocity even for the subgroup of the most accurate saccades. Van Beers (van Beers, 2007) observed variability of saccades in normal subjects and suggested that at least part of variability of accuracy-speed arises, in the brainstem regions in which the motor commands are generated.

In conclusion, the present study shows many abnormalities in saccades for DLB patients, i.e., increased latency even for tasks expected to promote short latency saccades, absence or reduced rates of express latency, low accuracy and peak velocity, and higher variability for all parameters. Such deficits occur for all four directions. These results reflect dysfunction of both cortical and subcortical oculomotor structures.

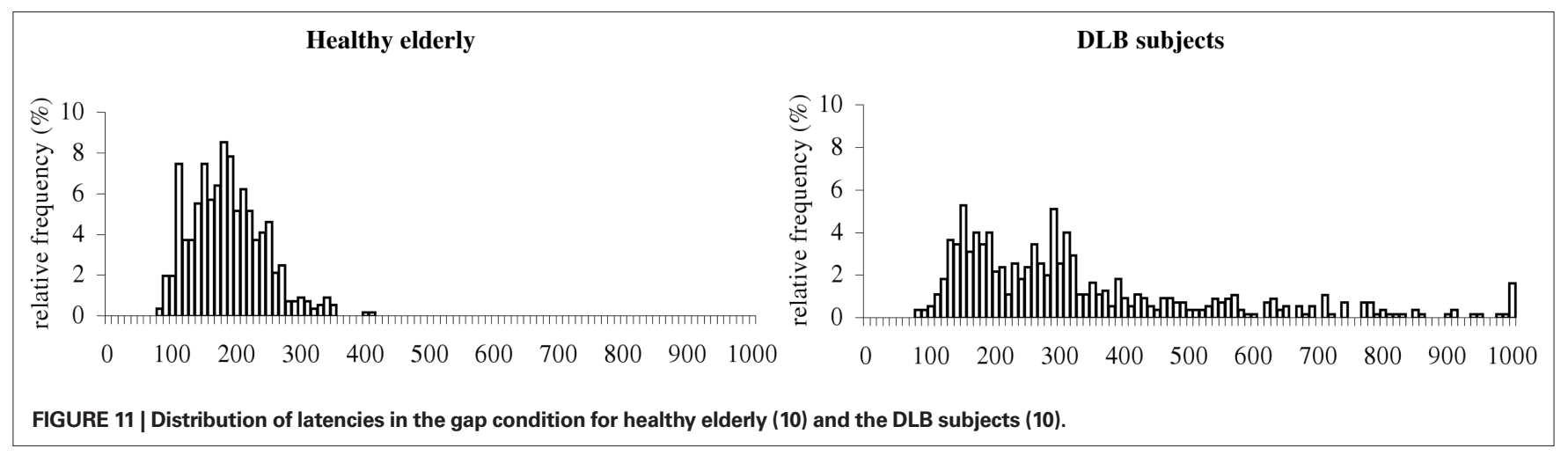




\section{REFERENCES}

Abel, L. A., Walterfang, M., Fietz, M., Bowman, E. A., and Velakoulis, D. (2009). Saccades in adult NiemannPick disease type $\mathrm{C}$ reflect frontal, brainstem, and biochemical deficits. Neurology 72, 1083-1086.

Armstrong, I. T., Chan, F., Riopelle, R. J., and Munoz, D. P. (2002). Control of saccades in Parkinson's disease. Brain Cogn. 49, 198-201.

Bell, A. H., Everling, S., and Munoz, D. P. (2000). Influence of stimulus eccentricity and direction on characteristics of pro- and antisaccades in non-human primates. J. Neurophysiol. 84, 2595-2604

Blekher, T., Johnson, S. A., Marshall, J., White, K., Hui, S., Weaver, M., Gray, J., Yee, R., Stout, J. C., Beristain, X., Wojcieszek, J., and Foroud, T. (2006). Saccades in presymptomatic and early stages of Huntington disease. Neurology 67, 394-399.

Boxer, A. L., Geschwind, M. D., Belfor, N., Gorno-Tempini, M. L., Schauer, G. F., Miller, B. L., Weiner, M. W., and Rosen, H. J. (2006). Patterns of brain atrophy that differentiate corticobasal degeneration syndrome from progressive supranuclear palsy. Arch. Neurol. 63, 81-86.

Carpenter, R. H., and Williams, M. L. (1995). Neural computation of log likelihood in control of saccadic eye movements. Nature 377, 59-62.

de Bruin, V. M., Lees, A. J., and Daniel, S. E. (1992). Diffuse Lewy body disease presenting with supranuclear gaze palsy, parkinsonism, and dementia: a case report. Mov. Disord. 7, 355-358.

Dorris, M. C., and Munoz, D. P. (1995). A neural correlate for the gap effect on saccadic reaction times in monkey. $J$. Neurophysiol. 73, 2558-2562.

Edelman, J. A., and Keller, E. L. (1996). Activity of visuomotor burst neurons in the superior colliculus accompanying express saccades. J. Neurophysiol. $76,908-926$.

Everling, S., Spantekow, A., Krappmann, P., and Flohr, H. (1998). Event-related potentials associated with correct and incorrect responses in a cued antisaccade task. Exp. Brain Res. 118, 27-34.

Findlay, J. M., and Walker, R. (1999). A model of saccade generation based on parallel processing and competitive inhibition. Behav. Brain Sci. 22, 661-674; discussion 674-721.

Fischer, B., Gezeck, S., and Huber, W. (1995). The three-loop model: a neural network for the generation of saccadic reaction times. Biol. Cybern. $72,185-196$

Folstein, M. F., Folstein, S. E., and McHugh, P. R. (1975). "Mini-mental state". A practical method for grading the cognitive state of patients for the clinician. J. Psychiatr. Res. 12, 189-198.

Garbutt, S., Matlin, A., Hellmuth, J., Schenk, A. K., Johnson, J. K., Rosen, H., Dean, D., Kramer, J., Neuhaus, J., Miller, B. L., Lisberger, S. G., and Boxer, A. L. (2008). Oculomotor function in frontotemporal lobar degeneration, related disorders and Alzheimer's disease. Brain 131 1268-1281.

Groschel, K., Hauser, T. K., Luft, A., Patronas, N., Dichgans, J., Litvan, I., and Schulz, J. B. (2004). Magnetic resonance imaging-based volumetry differentiates progressive supranuclear palsy from corticobasal degeneration. Neuroimage 21, 714-724.

Hanes, D. P., Patterson, W. F. 2nd, and Schall, J. D. (1998). Role of frontal eye fields in countermanding saccades: visual, movement, and fixation activity. J. Neurophysiol. 79, 817-834.

Heywood, S., and Churcher, J. (1980) Structure of the visual array and saccadic latency: implications for oculomotor control. Q. J. Exp. Psychol. 32, 335-341.

Hoehn, M. M., and Yahr, M. D. (1967). Parkinsonism: onset, progression and mortality. Neurology 17, 427-442

Horoupian, D. S., and Wasserstein, P. H. (1999). Alzheimer's disease pathology in motor cortex in dementia with Lewy bodies clinically mimicking corticobasal degeneration. Acta Neuropathol. 98 , 317-322.

Huaman, A. G., and Sharpe, J. A. (1993). Vertical saccades in senescence. Invest. Ophthalmol. Vis. Sci. 34, 2588-2595.

Irving, E. L., Steinbach, M. J., Lillakas, L., Babu, R. J., and Hutchings, N. (2006). Horizontal saccade dynamics across the human life span. Invest. Ophthalmol. Vis. Sci. 47, 2478-2484.

Isa, T., and Kobayashi, Y. (2004). Switching between cortical and subcortical sensorimotor pathways. Prog. Brain Res. 143, 299-305.

Israêl, L. (1996). "Evaluation de l'autonomie, les activités instrumentales de la vie quotidienne, IADL," in L'évaluation Clinique Standardisée En Psychiatrie, ed. J. D. Guelfi (Boulogne: éditions médicales Pierre Fabre, tome II), 477-480.

Kapoula, Z., Isotalo, E., Muri, R. M., Bucci, M. P., and Rivaud-Pechoux, S. (2001). Effects of transcranial magnetic stimulation of the posterior parietal cortex on saccades and vergence. Neuroreport 12, 4041-4046.

Kapoula, Z., Yang, Q., Coubard, O., Daunys, G., and Orssaud, C. (2004). Transcranial magnetic stimulation of the posterior parietal cortex delays the latency of both isolated and combined vergence-saccade movements in humans. Neurosci. Lett. 360, 95-99.

Kingstone, A., and Klein, R. M. (1993). Visual offsets facilitate saccadic latency: does predisengagement of visuospatial attention mediate this gap effect? J. Exp. Psychol. Hum. Percept. Perform. 19, 1251-1265.

Kurkin, S., Takeichi, N., Akao, T., Sato, F., Fukushima, J., Kaneko, C. R., and Fukushima, K. (2003). Neurons in the caudal frontal eye fields of monkeys signal three-dimensional tracking. Ann. N. Y. Acad. Sci. 1004 262-270.

Leigh, R. J., and Zee, D. S. (2006). The Neurology of Eye Movement. New York: Oxford University Press.

Mattis, S. (1988). Dementia Rating Scale Professional Manual. Odessa, FL: Psychological Assessment Resources.

McKeith, I. G., Dickson, D. W., Lowe, J., Emre, M., O'Brien, J. T., Feldman, H., Cummings, J., Duda, J. E., Lippa C., Perry, E. K., Aarsland, D., Arai, H., Ballard, C. G., Boeve, B., Burn, D. J., Costa, D., Del Ser, T., Dubois, B. Galasko, D., Gauthier, S., Goetz, C G., Gomez-Tortosa, E., Halliday, G., Hansen, L. A., Hardy, J., Iwatsubo, T. Kalaria, R. N., Kaufer, D., Kenny, R. A. Korczyn, A., Kosaka, K., Lee, V.M., Lees, A., Litvan, I., Londos, E., Lopez, O. L. Minoshima, S., Mizuno, Y., Molina, J.A. Mukaetova-Ladinska, E. B., Pasquier,F., Perry, R. H., Schulz, J. B., Trojanowski, J. Q., and Yamada, M. (2005). Diagnosis and management of dementia with Lewy bodies: third report of the DLB consortium. Neurology 65, 1863-1872.

Moschner, C., and Baloh, R. W. (1994). Age-related changes in visual tracking. J. Gerontol. 49, M235-M238.

Mosimann, U. P., Muri, R. M., Burn, D. J., Felblinger, J., O'Brien, J. T., and McKeith, I. G. (2005). Saccadic eye movement changes in Parkinson's disease dementia and dementia with Lewy bodies. Brain 128, 1267-1276.

Munoz, D. P., Broughton, J. R., Goldring, J.E., and Armstrong, I. T. (1998). Agerelated performance of human subjects on saccadic eye movement tasks. Exp. Brain Res. 121, 391-400.

Peltsch, A., Hemraj, A., Garcia, A., and Munoz,D.P. (2009).Age-related trends in saccade characteristics among the elderly. Neurobiol. Aging. doi:10.1016/j. neurobiolaging.2009.04.001

Pierrot-Deseilligny, C., Muri, R. M. Rivaud-Pechoux, S., Gaymard, B., and Ploner, C. J. (2002). Cortical control of spatial memory in humans: the visuooculomotor model. Ann. Neurol. 52, 10-19.

Pierrot-Deseilligny, C., Rivaud, S., Gaymard, B., Muri, R., and
Vermersch, A. I. (1995). Cortical control of saccades. Ann. Neurol. 37 557-567.

Pratt, J., Abrams, R. A., and Chasteen, A. L. (1997). Initiation and inhibition of saccadic eye movements in younger and older adults: an analysis of the gap effect. J. Gerontol. B. Psychol. Sci. Soc. Sci. 52, P103-P107.

Ratmanov, P. O., Napalkov, D. A., Bogdanov, R. R., Latanov, A. V., Turbina, L. G., and Shul'govskii, V. V. (2006). Effect of dopamine deficiency on the preparation of visually guided saccadic eye movements. $Z h$. Vyssh. Nerv. Deiat. Im. I P Pavlova 56, 590-596.

Reddi, B. A., and Carpenter, R. H. (2000). The influence of urgency on decision time. Nat. Neurosci. 3, 827-830.

Reuter-Lorenz, P. A., Hughes, H. C., and Fendrich, R. (1991). The reduction of saccadic latency by prior offset of the fixation point: an analysis of the gap effect. Percept. Psychophys. 49, 167-175.

Rolfs, M., and Vitu, F. (2007). On the limited role of target onset in the gap task: support for the motor-preparation hypothesis. J. Vis. 7, 1-20.

Roll, A., Wierzbicka, M. M., and Wolf, W. (1996). The "gap paradigm" leads to express-like saccadic reaction times in Parkinson's disease. Exp. Brain Res. $111,131-138$

Ross, L.E., and Ross, S.M. (1980). Saccade latency and warning signals: stimulus onset, offset, and change as warning events. Percept. Psychophys. 27, 251-257.

Ross, S. M., and Ross, L. E. (1981). Saccade latency and warning signals: effects of auditory and visual stimulus onset and offset. Percept. Psychophys. 29, 429-437.

Shafiq-Antonacci, R., Maruff, P., Masters, C., and Currie, J. (2003). Spectrum of saccade system function in Alzheimer disease. Arch. Neurol. 60 1272-1278.

Sharpe, J. A., and Zackon, D. H. (1987). Senescent saccades. Effects of aging on their accuracy, latency and velocity. Acta Otolaryngol. 104, 422-428.

Simard, M., and van Reekum, R. (2004). The acetylcholinesterase inhibitors for treatment of cognitive and behavioral symptoms in dementia with Lewy bodies. J. Neuropsychiatry Clin. Neurosci. 16, 409-425.

Sparks, D., Rohrer, W. H., and Zhang, Y. (2000). The role of the superior colliculus in saccade initiation: a study of express saccades and the gap effect. Vision Res. 40, 2763-2777.

Tedeschi, G., Di Costanzo, A., Allocca, S., Quattrone, A., Casucci, G., Russo, L., and Bonavita, V. (1989). Agedependent changes in visually guided 
saccadic eye movements. Funct. Neurol. 4, 363-367.

van Beers, R. J. (2007). The sources of variability in saccadic eye movements. J. Neurosci. 27, 8757-8770.

Van Opstal, A. J., and Van Gisbergen, J. A. (1989). A non-linear model for collicular spatial interactions underlying the metrical properties of electrically elicited saccades. Biol. Cybern. 60, 171-183.

van Stockum, S., MacAskill, M.,Anderson, T., and Dalrymple-Alford, J. (2008). Don't look now or look away: two sources of saccadic disinhibition in Parkinson's disease? Neuropsychologia 46, 3108-3115.

Warabi, T., Kase, M., and Kato, T. (1984). Effect of aging on the accuracy of visually guided saccadic eye movement. Ann. Neurol. 16, 449-454.

Yang, Q., Bucci, M. P., and Kapoula, Z. (2002). The latency of saccades, vergence, and combined eye move- ments in children and in adults. Invest. Ophthalmol. Vis. Sci. 43, 2939-2949.

Yang, Q., and Kapoula, Z. (2006). The control of vertical saccades in aged subjects. Exp. Brain Res. 171, 67-77.

Yang, Q., and Kapoula, Z. (2008). Aging does not affect the accuracy of vertical saccades nor the quality of their binocular coordination: a study of a special elderly group. Neurobiol. Aging 29, 622-638.

Yang, Q., Kapoula, Z., Debay, E., Coubard, O., Orssaud, C., and Samson, M. (2006). Prolongation of latency of horizontal saccades in elderly is distance and task specific. Vision Res. 46, 751-759.

Conflict of Interest Statement: In the present study, there are no any actual or potential conflicts including anyfi- nancial, personal or other relationships with other people or organizations within three years of beginning the work submitted that could inappropriately influence this work. Author's institution has no contracts relating to this research through which it or any other organization may stand to gain financially now or in the future. Authors or their institutions have no a financial interest in this work. This manuscript has not been published elsewhere and is not under review with another journal. The present investigation adhered to the tenets of the Declaration of Helsinki and was approved by the institutional human experimentation committee. Informed consent was obtained from all subjects after the nature of the procedure had been explained. All authors have reviewed the contents of the manuscript being submitted, approve of its contents and validate the accuracy of the data.
Received: 30 March 2010; paper pending published: 09 September 2010; accepted: 29 September 2010; published online: 22 November 2010.

Citation: Kapoula Z, Yang Q, Vernet M, Dieudonné B, Greffard S and Verny $M$ (2010) Spread deficits in initiation, speed and accuracy of horizontal and vertical automatic saccades in dementia with Lewy bodies. Front. Neur. 1:138. doi: 10.3389/ fneur. 2010.00138

This article was submitted to Frontiers in Dementia, a specialty of Frontiers in Neurology.

Copyright $\odot 2010$ Kapoula, Yang, Vernet, Dieudonné, Greffard and Verny. This is an open-access article subject to an exclusive license agreement between the authors and the Frontiers Research Foundation, which permits unrestricted use, distribution, and reproduction in any medium, provided the original authors and source are credited. 